\title{
Human Plasma Metabolites Measured with Different Liquid Chromatography/Mass Spectrometry (LC/MS) Platforms
}

\author{
Kelly H. Telu, Xinjian Yan, William E. Wallace, Stephen E. Stein, and Yamil Simón-Manso \\ National Institute of Standards and Technology, \\ Gaithersburg, MD 20899 \\ kelly.telu@nist.gov \\ xinjian.yan@nist.gov \\ william.wallace@nist.gov \\ stephen.stein@nist.gov \\ yamil.simon@nist.gov
}

Data DOI: http://dx.doi.org/10.18434/T4K88D

Key words: global metabolite profiling; human blood plasma; mass spectrometry; non-targeted metabolomics; Standard Reference Material.

Accepted: September 20, 2016

Published: September 23, 2016

http://dx.doi.org/10.6028/jres.121.022

\section{Summary}

Non-targeted global metabolite profiles of Standard Reference Material (SRM) 1950 Metabolites in Human Plasma were obtained on five different liquid chromatography/mass spectrometry (LC/MS) instrument combinations using identically prepared samples. Different instruments rendered significantly different metabolite profiles, however, the results were highly reproducible when working with one instrument. A full description of the measurements and data analysis have been previously published [1]. Presented here are the raw data on which Ref. [1] is based. For a further description of SRM 1950 and the general analysis of its chemical composition, see Refs. [2] and [3].

\section{Data Specifications}

\begin{tabular}{|l|l|}
\hline NIST Operating Unit & $\begin{array}{l}\text { Material Measurement Laboratory, Biomolecular Measurement Division, Mass Spectrometry Data } \\
\text { Center }\end{array}$ \\
\hline Format & Thermo Scientific .raw files; Agilent Technologies .d files \\
\hline Instrument & Thermo Scientific Orbitrap Elite; Agilent Technologies model 6530 Q-TOF \\
\hline Accessibility & $\begin{array}{l}\text { All datasets submitted to Journal of Research of NIST are publicly available. The set discussed here } \\
\text { can be found at: Telu_human_plasma_metabolites.zip }\end{array}$ \\
\hline License & NIST Licensing Policy \\
\hline Hash Value & SHA-256 Hash File \\
\hline
\end{tabular}




\section{Methods}

The liquid chromatography methods used were: conventional high-performance liquid chromatography (HPLC), ultra-high performance liquid chromatography (UHPLC), and nanoLC. The LC separations were coupled to two types of mass spectrometer: quadrupole-time of flight (QTOF), and orbitrap. The data provided includes the following combinations with replicate measurements: HPLC-orbitrap, nanoLC-orbitrap, HPLCQTOF, UHPLC-QTOF and nanoLC-QTOF. Reference [1] and its Supporting Information give a complete description of the sample preparation methods and the experimental conditions. Reference [1] also gives in spreadsheet form the authors' analysis of the data using three different software packages: the publicly available XCMS produced by the Scripps Research Institute, the proprietary Agilent Mass Profiler Professional ${ }^{1}$, and NIST’s own in-house software.

\section{Impact}

The raw data is provided to the scientific community as a benchmark for new measurement protocol development. It also allows for additional chemical compounds to be found in human blood plasma by the application of data analysis methods not employed by NIST especially those that will undoubtedly emerge in the future.

\section{References}

[1] Telu KH, Yan X, Wallace WE, Stein SE, Simón-Manso Y (2016) Analysis of human plasma metabolites across different liquid chromatography/mass spectrometry platforms: Cross-platform transferable chemical signatures. Rapid Commun Mass Spectrom 30(5):581-593. http://dx.doi.org/10.1002/rcm.7475

[2] Phinney KW, et al. (2013) Development of a Standard Reference Material for metabolomics research. Anal Chem 85(24):11732-11738. http://dx.doi.org/10.1021/ac402689t

[3] Simón-Manso Y, et al. (2013) Metabolite profiling of a NIST Standard Reference Material for human plasma (SRM 1950): GC-MS, LC-MS, NMR, and clinical laboratory analyses, libraries, and web-based resources. Anal Chem 85(24):11725-11731. http://dx.doi.org/10.1021/ac402503m

About the authors: Kelly Telu is a chemist working on metabolite identification in a variety of samples related to human health. XinJian (Eric) Yan is a chemist working on the development of new computer codes for the processing of complex LC/MS data. William Wallace is a materials research engineer specializing chemometric method development. Stephen Stein is a NIST Fellow with more than 35 years of experience in the interpretation of mass spectra. Yamil Simón is a chemist specializing in the analysis of complex mixtures by mass spectrometry. All authors are members of the NIST Mass Spectrometry Data Center. The National Institute of Standards and Technology is an agency of the U.S. Department of Commerce.

\footnotetext{
${ }^{1}$ Certain commercial equipment, instruments, or materials are identified in this paper to foster understanding. Such identification does not imply recommendation or endorsement by the National Institute of Standards and Technology, nor does it imply that the materials or equipment identified are necessarily the best available for the purpose.
} 